\title{
A Link in SFR and Morphology of Bulges
}

\author{
David B. Fisher \\ Department of Astronomy, University of Texas at Austin, Austin, TX 78712, USA \\ email: dbfisher@astro.as.utexas.edu
}

\begin{abstract}
We use Spitzer 3.6-8.0 $\mu \mathrm{m}$ color profiles to compare the radial structure of star formation of galaxies with pseudobulges and classical bulges. Galaxies structurally identified as having pseudobulges have higher central star formation rates than those of classical bulges. Further, galaxies identified as having classical bulges have remarkably regular star formation profiles. These observations support the picture of pseudobulge formation via secular evolution and classical bulges forming in mergers.
\end{abstract}

\section{Pseudobulges Are Forming Before Our Eyes}

Pseudobulges are "bulges" that we believe have formed through secular evolution, rather than mergers. We identify pseudobulges by the presence of disk structure (nuclear spirals, nuclear bars, flat stellar light ellipses) inside the bulge as outlined by Kormendy \& Kennicutt (2004); classical bulges have smooth, rounder isophotes compared to their outer disk. The color profiles of galaxies with classical bulges show a star forming outer disk with a sharp decline in star formation rates, toward the center of the galaxy. The inner $1.5 \mathrm{kpc}$ of $3.6-8.0 \mu \mathrm{m}$ profiles in classical bulges is constant and similar to elliptical galaxies. This is consistent with classical bulge formation reaching completion, followed by quiescent evolution. Pseudobulges, in general, show no such transition in star formation properties from the outer disk to the central pseudobulge. Bulges with nuclear bars, rings and spiral structure are forming stars just as vigorously as their outer disks.

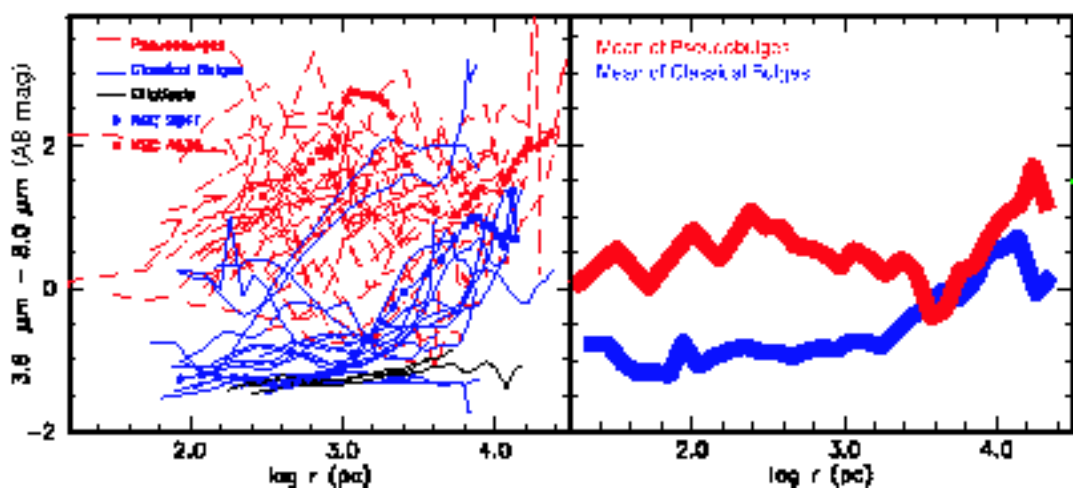

Figure 1. All 3.6-8.0 $\mu \mathrm{m}$ color profiles from Fisher (2006).

\section{References}

Kormendy, J., \& Kennicutt, .R.C. 2004, ARAA 42, 603.

Fisher, D.B. 2006, Ap. Lett. 642, L17. 